\title{
Retención de agua en musgos de páramo de los municipios de Siachoque, Toca y Pesca (Boyacá)
}

\section{Evaluation of the water retention in mosses of moor of Siachoque, Toca and Pesca (Boyaca)}
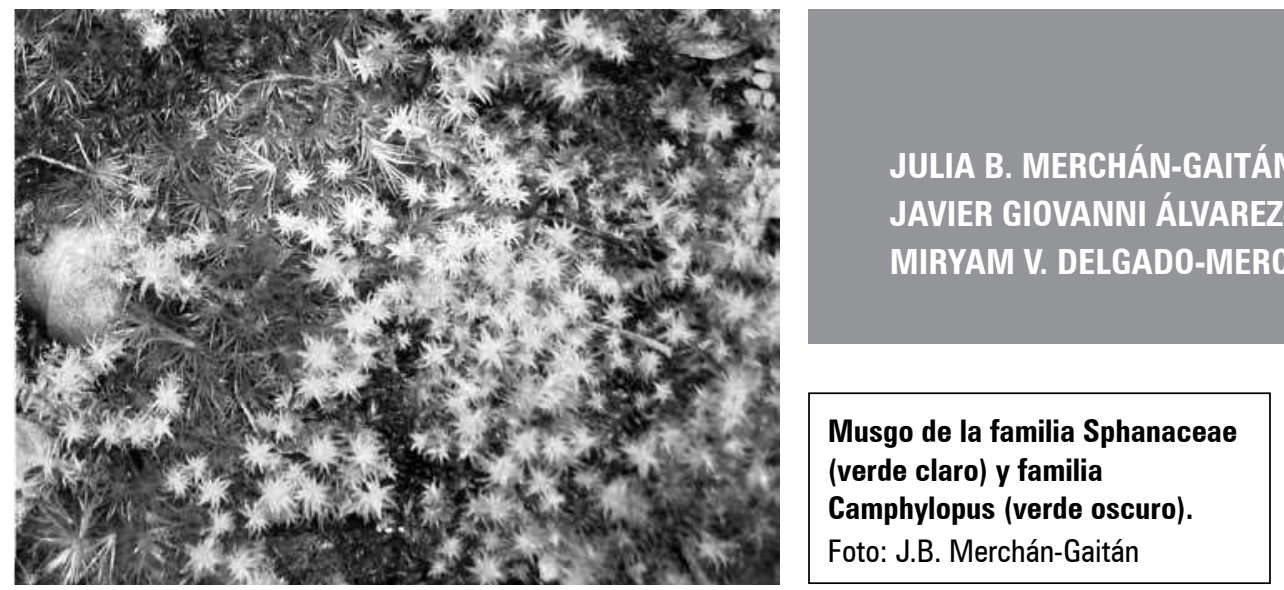

\section{RESUMEN}

Los musgos cumplen un papel importante en el ecosistema debido a su estructura morfológica para retener cantidades considerables de agua y mejorar los contenidos de humedad en el suelo. Sin embargo, el alto impacto del hombre en los páramos disminuye notablemente la población de los musgos y la conservación del recurso agua. Se evaluó la capacidad hídrica de muestras colectadas en los páramos de los municipios de Pesca, Siachoque y Toca (Boyacá) en diferentes sustratos (roca, suelo y corteza de árbol o epifitas). Se realizó un diseño completamente al azar y se determinó la capacidad hídrica en seco y en fresco. Se encontró que la familia con mayor número de muestras colectadas fue la Sphagnaceae en los páramos de los municipios de Pesca y Siachoque. Las muestras de musgos colectadas que poseen el mayor contenido de agua fueron las encontradas en el páramo del municipio de Pesca, mientras que las muestras colectadas en el municipio de Toca mostraron una gran variedad de familias de musgos (Leucomiaceae, Dicranaceae, Orthortrichaceae, Bartramyaceae) en menor población y con un menor contenido de agua. Además se presentó un mayor contenido de agua en las muestras colectadas del sustrato suelo que en las colectadas en roca y en epifitas, de ahí la importancia de las condiciones climáticas de cada una de las zonas en los diferentes municipios estudiados, pues afectan la retención de agua por parte de los musgos.

1 Facultad de Ciencias Agropecuarias, Grupo de Investigaciones Agrícolas, Universidad Pedagógica y Tecnológica de Colombia, Tunja (Colombia).

2 Facultad de Ciencias Básicas, Escuela de Biología, Universidad Pedagógica y Tecnológica de Colombia, Tunja (Colombia).

3 Autor para correspondencia. jgalvarezh@gmail.com 


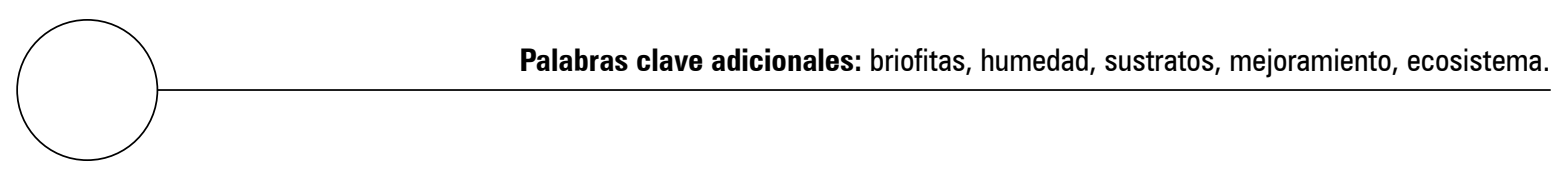

ABSTRACT

Mosses fulfill an important role in ecosystems, due to their morphologic structure that helps retain considerable amounts of water and improve soil moisture. The hydric capacity of samples collected in the moors of Pesca, Siachoque and Toca (Boyaca, Colombia) from different substrates (rock, soil and tree bark or epiphytes) was evaluated, using a completely randomized design. The most representative family was Sphagnaceae with the highest number of samples collected in the moors of Pesca and Siachoque. Moss samples collected with the highest water content were found in the moors of Pesca, whereas the samples collected in Toca had a great variety of moss families (Leucomiaceae, Dicranaceae, Orthortrichaceae, Bartramyaceae) with a smaller presence and lower water content. Also, the samples collected from the soil substrate presented higher water content than those collected from rock and epiphytes; therefore, indicating the importance of the climatic conditions of each of the zones in the studied municipalities, as they affect water retention by the mosses.

Additional keywords: briofits, humidity, substrates, improvement, ecosystem.

Fecha de recepción: 26-05-2011

Aprobado para publicación: 21-11-2011

INTRODUCCIÓN

La abundancia de agua en los ambientes donde se encuentran los musgos es bien conocida. Esta es normalmente visible en la superficie del suelo debido a la alta capacidad de retención de humedad que poseen las diferentes especies vegetales que están presentes (vasculares y no vasculares). Los musgos constituyen reservorios de agua cuya importancia en la regulación del escurrimiento está en relación con la proporción de las áreas cubiertas en el contexto de una cuenca hídrica, esas acumulaciones de agua son atribuibles a las propiedades físicas del Sphagnum (Tomotsugu et al., 2006).

El género Sphagnum comprende numerosas especies de musgos Sphagnaceae predominantes en la mayoría de páramos o pantanos (Torres y Esquivel, 1998). Deben su capacidad para absorber grandes cantidades de agua a la conformación y estructura celular de sus tejidos. El tejido cortical de los tallos está formado por células grandes e hidrófilas, comunicadas entre sí y con el medio externo a través de poros (Reese, 1978).

Las hojas de los musgos gametofitos no tienen tejido mesófilo, estomas, o venas relacionadas, como las hojas de las plantas más complejas Stern (2003), por consiguiente no poseen parénquima. Las hojas muy reducidas, están formadas por un solo estrato de células, también de gran tamaño. Gran parte de las células de las hojas, vacías de plasma, son no clorofílicas, y cumplen la función de almacenar el agua disponible en el medio exterior (Bernal et al., 2006).

En consecuencia, estos tejidos hidrófilos de los musgos experimentan variaciones volumétricas celulares muy significativas a lo largo del año, de acuerdo con la tasa de evapotranspiración y a la disponibilidad de agua, procedente funda- 
mentalmente de las precipitaciones locales, en el caso de los túrbales (ecosistemas de humedales que acumulan turba en profundidad) elevados; la mayor parte de los musgos utilizan el agua como medio para poderse reproducir (Iturraspe y Roig, 2000).

La enorme capacidad de retención de agua de los túrbales se debe a las propiedades absorbentes del musgo Sphagnum. La importancia de estos túrbales en zonas ubicadas a gran altitud sobre el nivel del mar trasciende su propia dimensión. Por consiguiente, si estos túrbales se destruyen, la perturbación hidrológica local puede resultar catastrófica (Correa, 1987). Así mismo, su rol ecológico es significativo para el funcionamiento del ecosistema como agentes activos de retención de agua, colonizadores y estabilizadores del suelo. Según Avendaño y Torres (2007) la turba está constituida por restos vegetales principalmente de musgos del género Sphagnum y de algunas gramíneas, acumuladas y comprimidas. La turba blanca (del musgo Sphagnum) se emplea, principalmente, como combustible y aditivo de suelos (Steubing et al., 2001).

El musgo Sphagnum ha sido usado efectivamente como agente para filtración y tratamiento de aguas servidas y efluentes de industrias con descargas ácidas y tóxicas, con alto contenido de metales pesados y substancias orgánicas, tales como aceites, detergentes o tinturas (Matteri, 1998).

La estructura de los microorganismos del suelo también puede cambiar con el cambio de temperatura y de la humedad en los musgos, lo que puede facilitar la evaluación del efecto a largo plazo del cambio climático sobre la descomposición de turbales (Nakatsubo et al., 1997).

El objetivo de este trabajo fue determinar el contenido de humedad de los musgos colectados en tres municipios del departamento de Boyacá, en especial turbales de Sphagnum, con el fin de conocer su potencial como almacenadores de agua.

\section{MATERIALES Y MÉTODOS}

\section{Localización}

El presente trabajo de investigación se llevó a cabo en tres localidades las cuales tienen las siguientes condiciones: el primer sitio de muestreo esta ubicado en el páramo de Siachoque, en el municipio del mismo nombre, a una altitud de $4.100 \mathrm{msnm}$ con una temperatura media de $6^{\circ} \mathrm{C}$, posee una humedad relativa mínima de $38 \%$ y máxima de $70 \%$, este municipio tiene una extensión de $125 \mathrm{~km}$ y es atravesado por los ríos Cormechoque, Tocavita y Turga (Correa, 1987). El municipio de Toca en la vereda de la Chorrera situada a una altitud $2.950 \mathrm{msnm}$, posee una humedad relativa mínima de $37 \%$ y máxima de $63 \%$ y su temperatura media es de $13^{\circ} \mathrm{C}$. El municipio de Pesca se encuentra ubicado en la cordillera oriental, a una altitud de $3.910 \mathrm{msnm}$, posee una humedad relativa mínima de $37 \%$ y máxima del $78 \%$, su temperatura media es de $7^{\circ} \mathrm{C}$, cuenta con ecosistemas estratégicos, como el páramo y bosques, complementados con actividades productivas agropecuarias en la vereda Paso Grande (Correa, 1987).

\section{Diseño experimental y métodos}

Para la colecta de los musgos en los tres páramos se utilizó un diseño completamente al azar, los tratamientos corresponden a los tres municipios (Siachoque, Pesca y Toca) y 12 muestras por tratamiento, equivalentes a las repeticiones, tomando transectos de $2 \mathrm{~m}$ de ancho $\times 10 \mathrm{~m}$ de largo, evaluando un total de 36 unidades experimentales. La variable medida fue el contenido hídrico $(\mathrm{CH})$, calculado a partir de la cantidad de agua que retienen los musgos en función de su masa en diferentes sustratos, colectando muestras en cada sitio entre las 8 y 10 a.m. en los meses de marzo y abril no lluviosos.

Las muestras se colectaron con una pala de jardinería de medida $10 \times 5 \mathrm{~cm}$ de longitud en diferentes sustratos (roca, suelo y corteza de árbol), 
las muestras colectadas se pesaron en fresco en el mismo lugar, con una balanza electrónica Acculab VIC 612 de 0,01 g de precisión, luego se introdujeron en bolsas plásticas y posteriormente en bolsas de papel para ser transportadas al laboratorio, el material colectado se seco a temperatura de $75^{\circ} \mathrm{C}$ durante $48 \mathrm{~h}$ en mufla. Después de que las muestras fueron secadas, se prosiguió a pesarlas nuevamente, el peso de los musgos en fresco se restó al peso de los musgos secos y el resultado de esta diferencia es el contenido de humedad que poseen las diferentes familias de musgos colectadas en los páramos de Toca, Siachoque y Pesca, metodología recomendada por Steubing et al. (2001).

Luego, se procedió a hacer la identificación de las muestras en el laboratorio de Botánica de la UPTC con la ayuda de un estereoscopio, microscopio óptico y clave para musgos, sugerido por Churchill y Linares (1995). Los ejemplares reposan en el herbario de la UPTC debidamente determinados y enumerados.

\section{Análisis de la información}

Se realizaron análisis de varianza (Anova) con el fin de determinar la existencia de diferencias estadísticas entre las diferentes zonas y sustratos evaluados, así mismo mediante la prueba de comparación de Tukey al 0,05\% se compararon los tratamientos, para lo cual se utilizó el software SAS v. 8.1e (Cary, N.C).

\section{RESULTADOS Y DISCUSIÓN}

\section{Familias de musgos y descripción}

Como resultado de los muestreos realizados se encontró que el mayor número de muestras pertenece a la familia Sphagnaceae, genero Sphagnum con un total de 25 ejemplares (figura 1), estas representan un $67 \%$ del total de las plantas colectadas, seguida por la familia Ditrichaceae, genero Ditrichum con un $14 \%$.

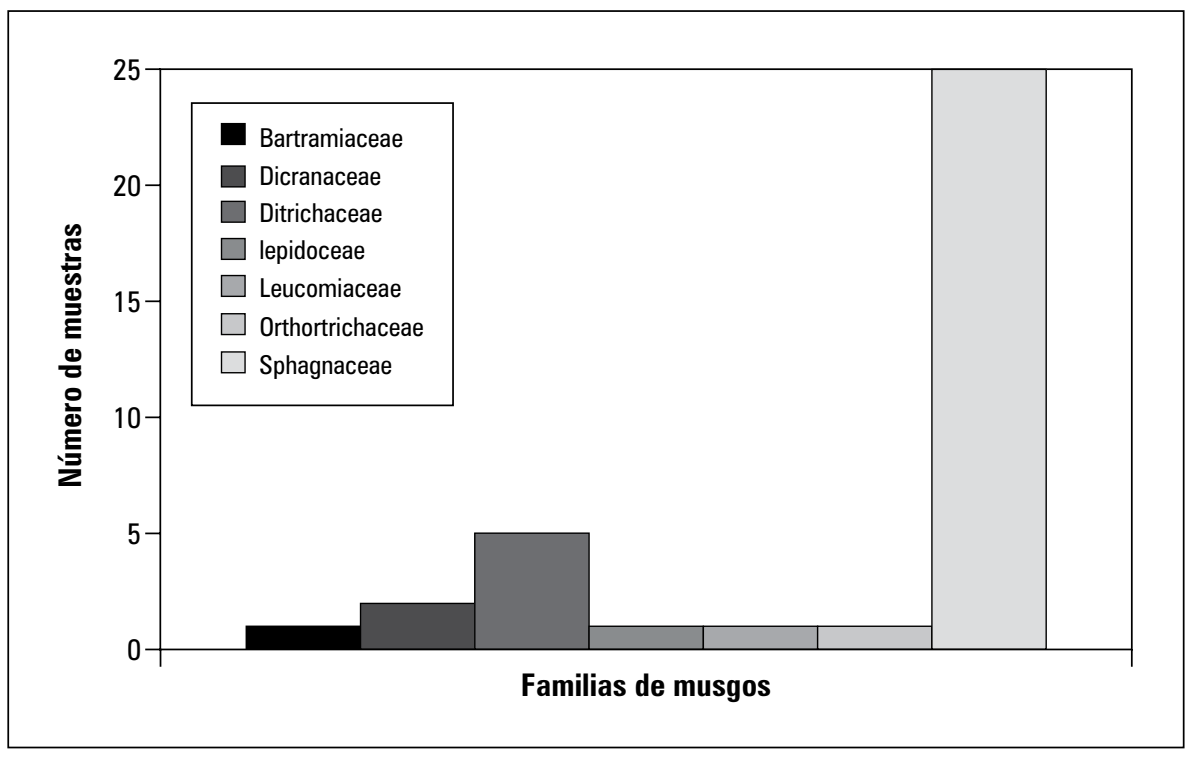

Figura 1. Número de muestras de musgos encontradas y clasificadas por familia para los transectos realizados en los municipios de Siachoque, Toca y Pesca. 
La familia Ditrichaceae, genero Ditrichum con tallos erectos simples, varias veces ramificados, hojas apretadas a distantes, células mediales subcuadradas hasta irregularmente corto-rectangulares, lisas hasta mamilosas o papilosas, células inferiores y básales oblongo-rectangulares, usualmente lisas (Churchill y Linares, 1995).

El 29\% restante de las muestras encontradas pertenece a las familias de Leucomiaceae (Leucomium), Dicranaceae (Campylopus), Orthortrichaceae (Macromitrium), Bartramyaceae (Bartramia) y a la familia Lepidoziaceae (lepidozia) que pertenece a las hepáticas, las cuales suelen confundirse con los musgos, debido a que poseen un caulidio (eje) fijado también por rizoides al sustrato, además a lo largo del caulidio poseen unas pequeñas hojas o filidios (Delgadillo y Cárdenas, 1990). Esta diferencia en número se da debido a que las muestras se colectaron en partes de mucha humedad y de mayor altura, que son los sitios donde mejor se desarrollan las Sphagnaceae.

La familia Leucomiaceae, género Leucomium contiene plantas pleurocárpicas de $7 \mathrm{~cm}$ de largo, verde pálidas a amarillo doradas, tallos postrados irregularmente pinnados Frahm (1991), hojas ovadolanceoladas de $3 \mathrm{~mm}$ de largo; falcado-secundas y cóncavas; ápice cetáceo, margen entera a finamente serrada distalmente, sin costa (Sastre et al., 1986); células lisas, las mediales largo-fusiformes y basales anchamente fusiformes, hábitat y distribución: crece sobre bases de árboles y madera en descomposición en forma de tapete liso dentro de bosques en ambientes parcialmente oscuros y húmedos entre los 3.100 y los 3.200 msnm (Álvaro et al., 2006).

La familia de las Dicranaceae, género Campylopus presenta gran variación especialmente en su tamaño, pero guarda características similares con otros géneros en cuanto a la morfología de sus hojas; por ejemplo, todas las Campylopus presentan una gran costa que ocupa más de la mitad de toda la hoja, esto hace fácil reconocerlas (Churchill y Linares, 1995).
La familia Orthotrichaceae, género Macromitrium con tallos primarios erectos o rastreros, con tallos secundarios y ramas erectas o raramente subpéndulas, poco a muy ramificados; hojas comúnmente crispadas, lineo-lanceoladas a oblongo-lanceoladas, lisas ocasionalmente rugosas, plegadas, ápices agudos hasta acuminados. Células de la lámina con paredes gruesas, células superiores y mediales isodiamétricas hasta alargadas, lisas, mamilosas o papilosas, células basales alargadas (Churchill y Linares, 1995).

La familia Bartramyaceae, género Bartramia con tallos erectos de 2 a $7 \mathrm{~cm}$ de largo, poco a muy ramificados mediante brotes de innovación; hojas conspicuamente dispuestas en 5 filas o más, comúnmente de forma espiralada; células superiores y mediales subcuadradas (Churchill y Linares, 1995).

En cuanto al contenido de agua que retienen los musgos, se observó un mayor contenido significativo en las muestras colectadas en Pesca (164 g), seguidas por las de Siachoque (156 g) como lo indica la figura 2. Esto es debido a que dichos municipios las colecciones se tomaron del sustrato (suelo), el cual presenta una mayor cantidad de agua disponible para el desarrollo de los musgos en comparación con los otros sustratos evaluados.

Los musgos logran vivir con éxito en las zonas xerofíticas gracias a su adaptación para contener humedad y retenerla, esto permite que en épocas de sequía permanezcan húmedos y en sequías prolongadas se mantengan en un periodo latente de modo que cuando lleguen las lluvias reverdezcan nuevamente de acuerdo con lo indicado por Correa (1987).

En el municipio de Toca se colectaron mas musgos del sustrato suelo que de los sustratos de roca y de corteza de árbol (figura 3); estos sustratos presentan una menor disponibilidad de agua, por lo que el musgo va a presentar una menor acumulación de humedad ya que no puede capturar buenas cantidades de agua del medio circundante (Equihua et al., 2001). 


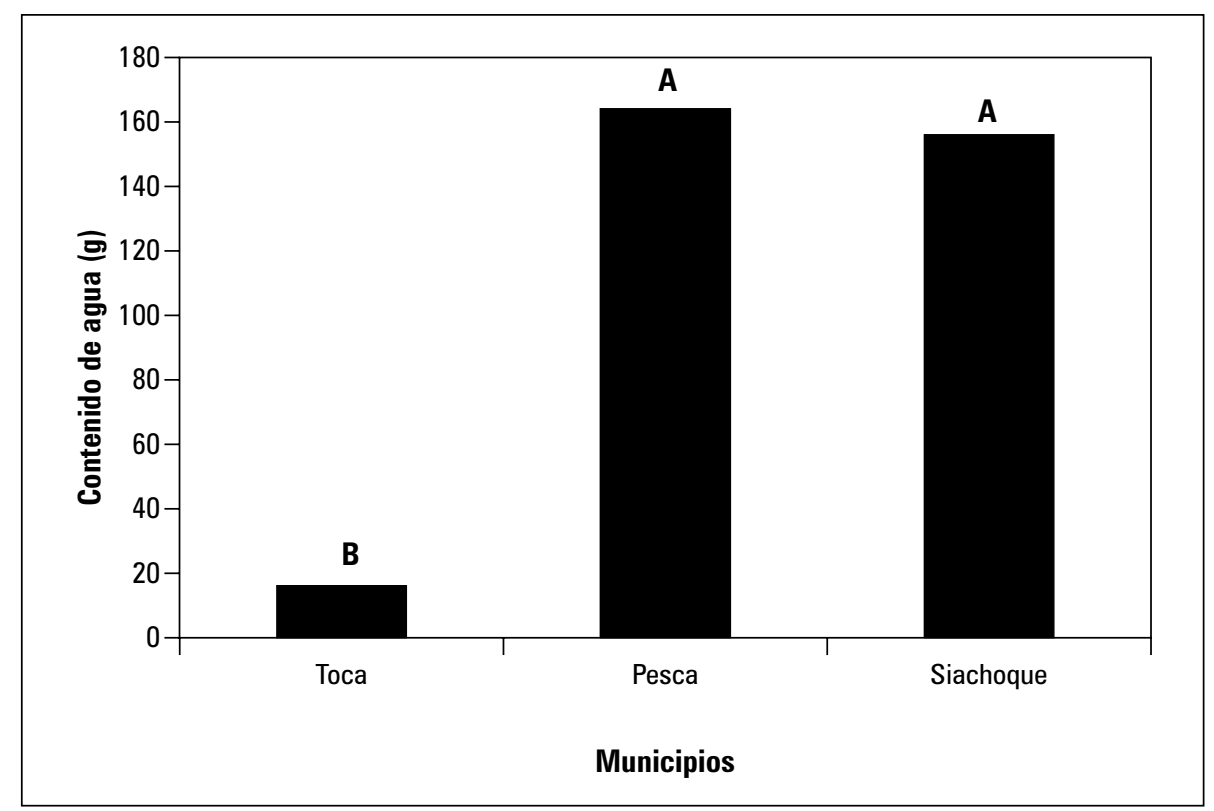

Figura 2. Contenido de agua promedio de los musgos encontrados en los municipios de Siachoque, Toca y Pesca. Promedios con letras distintas indican diferencia significativa según la prueba de Tukey $(P \leq 0,05)$.

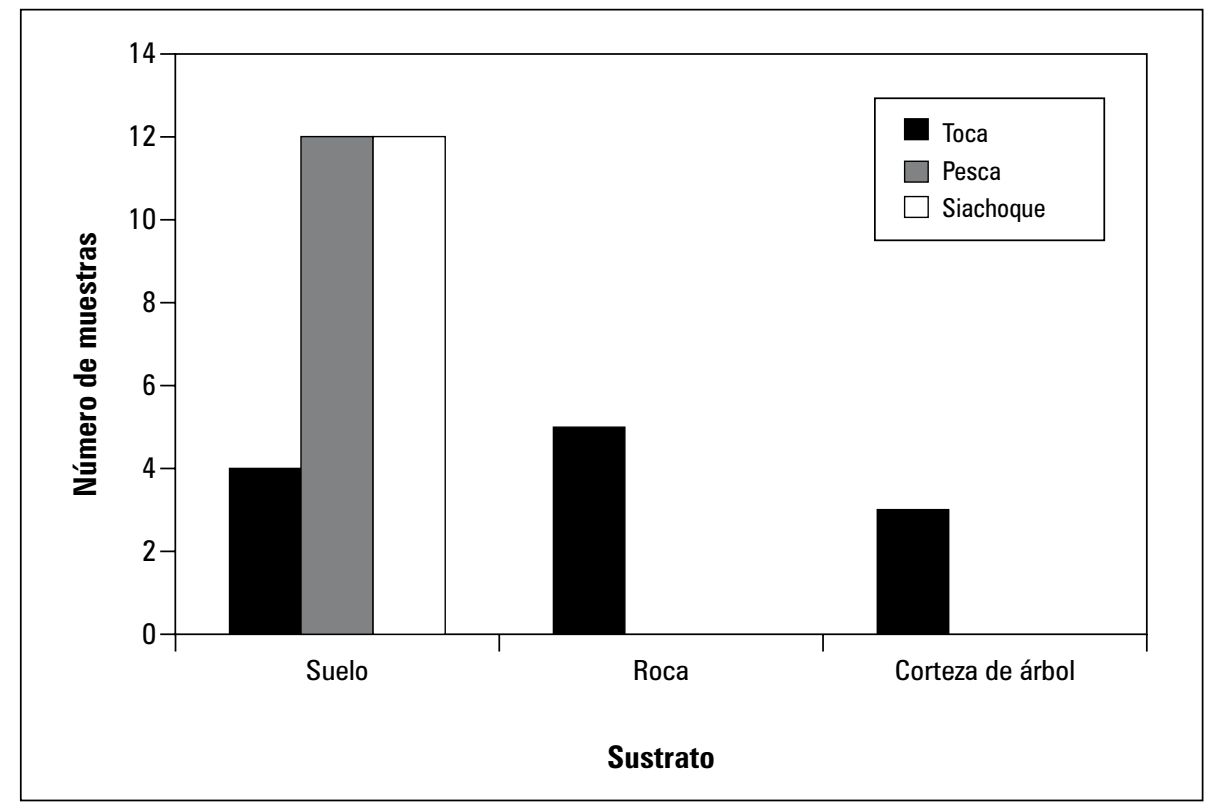

Figura 3. Número de muestras de musgos encontradas en cada uno los sustratos para los transectos realizados en los municipios de Siachoque, Toca y Pesca.

La familia de mayor contenido de agua encontrada en este estudio es la Sphagnaceae sphagnum (figura 4), esto es debido a la fácil captación de agua de esta planta, por su morfología y estructura celular grande e hidrofílica, que la hace absorben- te (Takeshi y Hiroshi, 2006). Con respecto a las propiedades funcionales del Sphagnum en estudios realizados en otros ecosistemas, estos mostraron los mejores resultados de capacidad de absorción y retención de agua (Villarroel et al., 2003). 


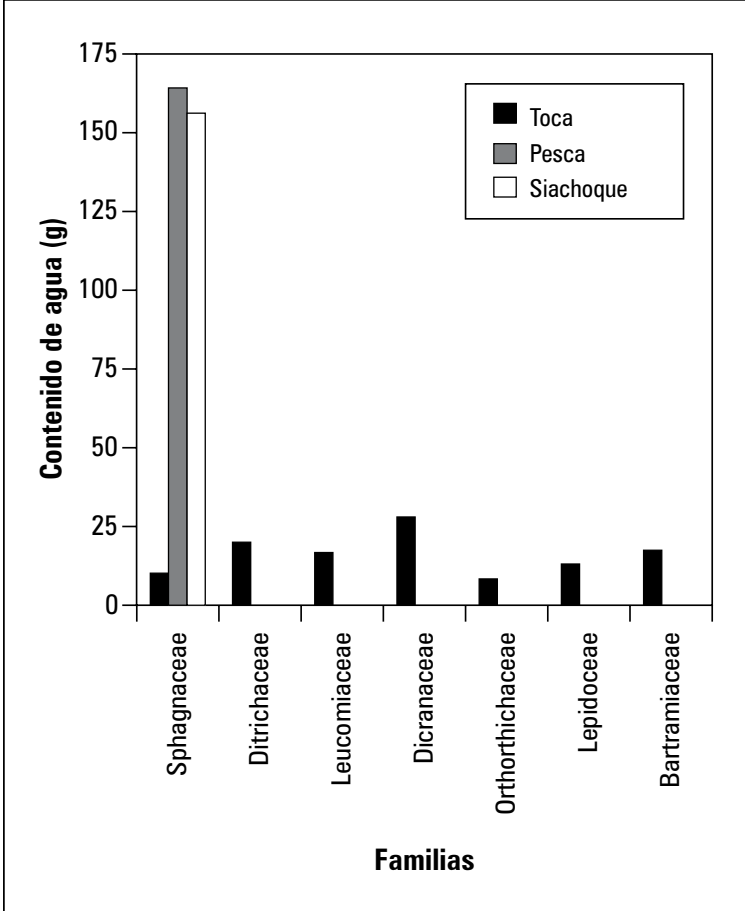

Figura 4. Contenido de agua de musgos clasificados por familia encontrados en transectos realizados en los municipios de Siachoque, Toca y Pesca.

Las plantas Sphagnaceae son robustas de varios colores, con mayor presencia del tono verde oscuro y unas veces más brillantes que otras; cabezuelas con ramas gruesas, romas y filidios escuarrosos. Yema terminal visible pero no conspicua. Fascículos formados por dos ramas divergentes largas y sinuosas y dos péndulas más finas y sin filidios escuarrosos. Membrana de los hialocistos hacia el ápice por lo general reabsorbida en ambas caras. Los hialocistos de la base presentan poros que pueden ser muy grandes y adoptar forma de lagunas de membrana. Borde en general estrecho (Munín y Fuertes, 2001). En cuanto a su ecología (figura 5), estas suelen formar césped más o menos extenso, constituyendo alfombras de vistosos colores con encharcamientos abundantes en zonas usualmente expuestas. Según Munín y Fuertes (2001), en cuanto a la hidromorfía del sustrato presenta un amplio rango de posibilidades por la presencia de ríos y riachuelos (Frahm y Newton, 2005). Puede aparecer en conjunto con una turba profunda más o menos compacta soportando cierta sequía superficial y con aportes minerales por movilidad del agua, o en zonas muy expuestas de suelo poco profundo pero muy encharcado. Para las demás familias, se presentan contenidos de agua mucho más bajos, que no superan $25 \mathrm{~mL}$ por muestra, lo que puede ser ocasionado por una estructura celular más pequeña y menos hidrofílica (Narváez et al., 2002).

Se colectó un mayor número de muestras de suelo en los municipios de Pesca y Siachoque (figura 4), debido a la poca presencia de roca, siendo un campo abierto de pocos árboles, mientras que en el municipio de Toca, es de bosque frondoso y rocoso, por esta razón se colectaron más muestras cortícolas (que crecen sobre la corteza de los árboles) y rupícolas (que se crían en roca) (Marsh y Koerner, 1972).

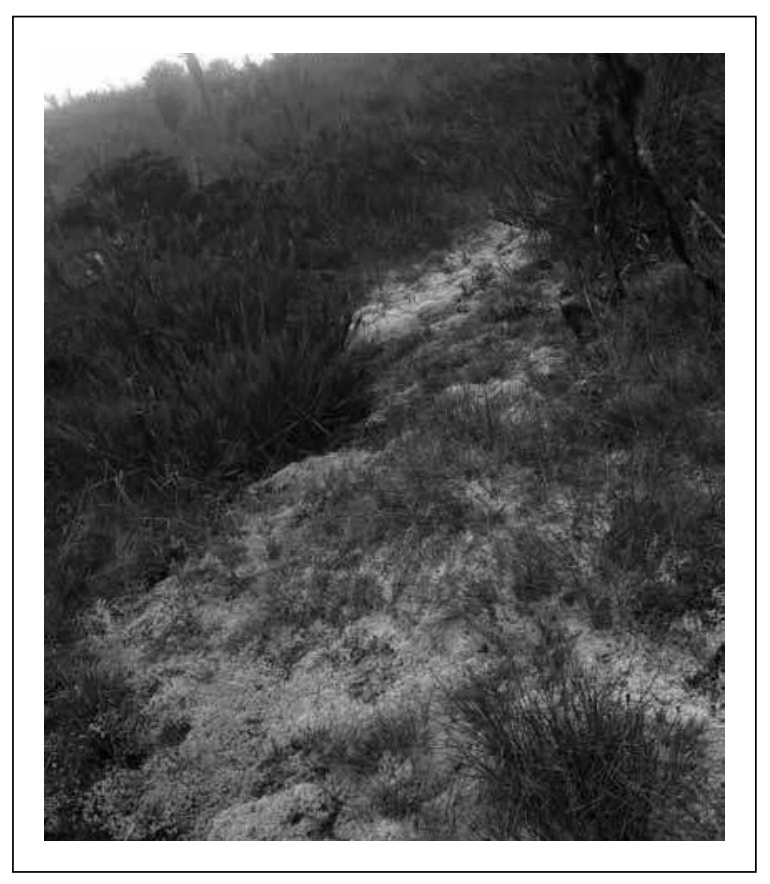

Figura 5. Plantas de Sphagnaceae sphagnum encontradas en la zona de estudio evaluada.

\section{CONCLUSIONES}

El mayor número de muestras colectadas pertenece a la familia Sphagnaceae. La familia de musgos que más contenido de agua presenta es 
la Sphagnaceae. El municipio donde se encontró la mayor diversidad de musgos es el de Toca. Los musgos de mayor contenido de agua se presen- tan en el municipio de Pesca. En general, el suelo es el sustrato que más alberga musgos Sphagnaceae sphagnum.

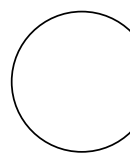

Álvarez, W., A. Díaz y J. Zabala. 2006. Flórula de la reserva forestal protectora "El Malmo" Tunja (BoyacáColombia). UPTC, Tunja, Colombia.

Avendaño, K. y J. Torres. 2007. Los musgos (Bryophyta) de la región de Santa María-Boyacá (Colombia). Caldasia 29(1), 59-71.

Bernal, E. S. Celis, X. Galíndez, C. Moratto, J. Sánchez y D. García. 2006. Microflora cultivable y endomicorrizas obtenidas en hojarasca de bosque (Páramo Guerrero, Finca Puente de Tierra) Zipaquirá, Colombia. Acta Biol. Colomb. 11(2), 125-130.

Churchill, S. y E. Linares. 1995. Pródromus Bryologiae Novo-Granatensis. Introducción a la Flora de Musgos de Colombia. Instituto de Ciencias Naturales, Universidad Nacional de Colombia, Bogotá.

Correa, R.C. 1987. Monografías de los pueblos de Boyacá Tomos I y II. Publicaciones de la Academia Boyacense de Historia. Editorial ABC, Bogotá.

Delgadillo, M. y A. Cárdenas. 1990. Manual de briofitas. $2^{a}$ ed. Instituto de Biología, UNAM, México, D.F.

Equihua, C. A. Newton, A. García, E. De Luna y R. Gradstein. 2001. New moss records for Mexico from the Lacandona tropical rain forest, Chiapas. The Bryologist 104(4), 602-606.

Frahm, J. P. y A. Newton. 2005. A new contribution to the moss flora of Dominican Amber. The Bryologist 108(4), 526-536.

Frahm, J.P. 1991. Dicranaceae. Campylopodioidae, Paraleucobryoideae. Flora Neotropica Monograph 54, 1-128.

Iturraspe, R. y C. Roig. 2000. Aspectos hidrológicos de turberas de Sphagnum de tierra del fuego -Argentina. pp. 85-93. En: Coronato, A. y C. Roig (eds.). Conservación de ecosistemas a nivel mundial con énfasis en las turberas de Tierra del Fuego. Ushuaia, Argentina.

Marsh, W. M. y J. Koerner. 1972. Role of moss in slope formation. Ecology 53(3), 489-493.

Matteri, C. 1998. Diversidad briológica (o sobre cómo y por qué proteger los musgos). Ciencia Hoy 8(46), 30-36.

\section{REFERENCIAS BIBLIOGRÁFICAS}

Munín, E. y E. Fuertes. 2001. Sphagnum section Squarrosa (Sphagnaceae, Musci) en España, Rev. Bot. Complutensis 25, 271-287.

Nakatsubo, T., M. Uchida, T. Horikoshi y K. Nakane. 1997. Comparative study of the mass loss rate of moss litter in boreal and subalpine forests in relation to temperature. Ecolog. Res. 12(1), 47-54.

Narváez, P., J. Jerez e I. Sastre de Jesús. 2002. Variación en la composición de especies de musgos sobre sustratos arbóreos en Añangu, selva amazónica (Provincia de Napo, Ecuador). En: Rangel, C., J. Aguirre y C. Andrade (eds.). Libro de Resúmenes VIII Congreso Latinoamericano de Botánica. II Congreso Colombiano de Botánica. Universidad Nacional de Colombia. Bogotá.

Reese, W.D. 1978. The genus Syrrhopodon in the Americas I. The limbate species. The Bryologist 81, 189-225.

Sastre de Jesús, I., S. Churchill y M. Escobar. 1986. Catálogo de musgos del Departamento de Antioquia. Colombia. Rev. Actual. Biol. (15), 77-94.

Stern, K. R. 2003. Introductory plant biology. $9^{\text {th }}$ ed. McGraw Hill, Boston, MA.

Steubing, L., A. Godoy y M. Alberdi. 2001. Métodos de ecología vegetal. Universitaria S.A., Santiago de Chile.

Takeshi, U. y K. Hiroshi. 2006. Photosynthetic response of the arctic semi-aquatic moss Calliergon giganteum to water content. Tokyo. Aquat Bot. 85(3), 241-243.

Tomotsugu, Y. U. Shin-Ichi y Y. Kazuo. 2006. Water balance and water movement in unsaturated zones of Sphagnum hummocks in Fuhrengawa Mire. Hokkaido, Japan. J. Hydrol. 319(1-4), 312-327.

Torres, C. y H. Esquivel. 1998. Determinación, descripción e importancia ecológica de los musgos de la reserva natural de Acaime (Municipio de Salento-Quindío). Rev. Asoc. Colomb. Ciencias Biol. 10(1-2), 75-81.

Villarroel, M., C. Acevedo, E. Yáñez y B. Biolley. 2003. Propiedades funcionales de la fibra del musgo Sphagnum magellanicum y su utilización en la formulación de productos de panadería. Chile. Rev. Alan. (53), 4-6. 